\title{
POWER PROCESSING CIRCUITS FOR ELECTROMAGNETIC, ELECTROSTATIC AND PIEZOELECTRIC INERTIAL ENERGY SCAVENGERS
}

\author{
P. D. Mitcheson, T. C. Green and E. M. Yeatman
}

Department of Electrical and Electronic Engineering, Imperial College London, SW7 2AZ, U.K.

\begin{abstract}
Inertial energy scavengers are self-contained devices which generate power from ambient motion, by electrically damping the internal motion of a suspended proof mass. There are significant challenges in converting the power generated from such devices to useable form, particularly in micro-engineered variants. This paper examines this power conversion requirement for each of the cases of electromagnetic, electrostatic and piezo-electric transduction, and presents new circuit approaches for the first two of these.
\end{abstract}

\section{INTRODUCTION}

Micro-scale energy-scavenging devices have been increasingly reported in the research literature during the last 10 years or so (Tharner and Paradiso 2004), and the number of active research groups in the field has grown steadily during that time. This interest is driven by a desire to eliminate primary batteries in mobile and other portable low power electronic devices, particularly wireless sensor nodes. Potential applications of such sensors include body sensor networks for health and fitness, security monitoring of indoor and outdoor spaces, and machine diagnostics. Large reductions in the power requirements of sensor electronics, including wireless communications, have not only made such networks more attractive but have made scavenging approaches to powering them more realistic.

Motion and vibration are attractive sources of energy for scavenging, particularly where ambient light and substantial temperature differences are not available. Some motion scavengers, such as heel strike generators (Tharner and Paradiso 2004), exploit the relative motion between two anchor points. Most, particularly at the $\mathrm{mm}$ size scale, have only a single anchor point, and extract energy from the relative motion between this point and an internal proof mass typically mounted on a spring suspension within a frame. These we refer to as inertial generators. When the frame is accelerated, causing relative displacement between the frame and proof mass, energy is extracted from the mechanical system by an electric damping mechanism which may be electromagnetic (typically a coil and permanent magnet) (Williams and Yates, 1995), electrostatic (a variable capacitor) (Meninger et al., 2001) or piezoelectric (normally a cantilever bimorph structure) (Roundy et al., 2003). Most of the reported generators are based around resonant mass-spring systems, although for some applications (particularly generators designed to power medical devices) non-resonant systems can achieve higher power densities (Mitcheson et al., 2004b).

The majority of reported work to date has concentrated on the design and fabrication of the massspring system and the transducer, with many groups using MEMS technology for fabrication. Testing has normally been achieved by measuring dissipated power in a resistor. Little work has been reported on the power processing electronics, one of the functions of which is to form the interface between the transducer and the load; load circuitry requires a steady DC voltage rail and the transducer of an inertial generator does not produce a stable voltage.

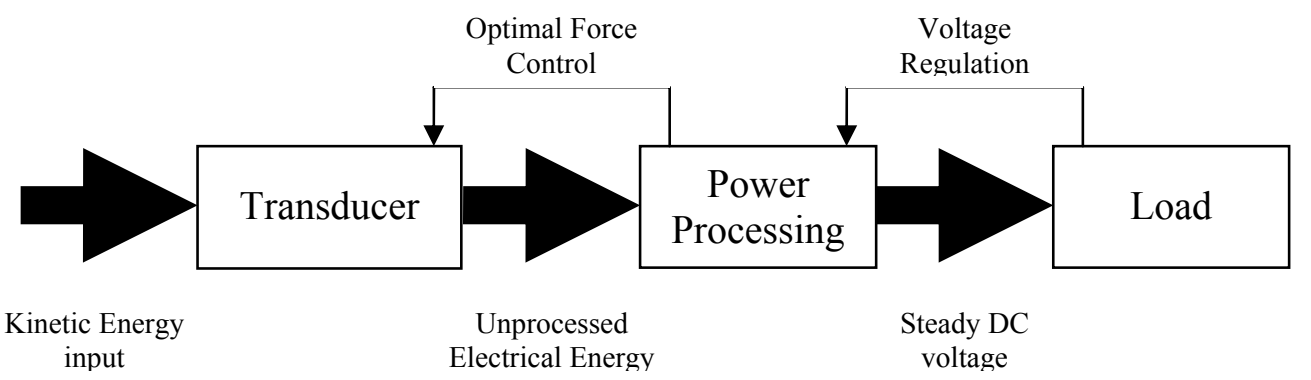

Figure 1 Block diagram of micro-generator system with power electronics 


\section{P. D. Mitcheson, T. C. Green and E. M. Yeatman, "Power Processing Circuits ..."}

The power processing electronics in a micro-generator must perform a second critical function in addition to providing a stable DC power source. There are limits on power density of an inertial energy scavenger which are primarily dependent upon the size of the generator, the motion which drives the generator frame and the architecture (Mitcheson et al., 2004a). In order to achieve the highest possible power density under a given operating condition, it is necessary that the damping force is set to an optimal value, because of a trade-off between the force provided by the damper and the size of the relative motion between the mass and the frame. This value of damping is the one which achieves maximum energy conversion, and thus when the transducer is operated to achieve high power densities, the electrical requirements of the damper and its characteristics are set by the need for this optimal damping force rather than simply by the electrical requirements of the load. The damping characteristics of transducer types are typically varied as follows:

- Electromagnetic - the damping force can be altered by the resistance of the load connected to the coil.

- Electrostatic - the damping force can be set by the electric field between the capacitor electrodes.

- Piezoelectric - the damping force can be altered by the impedance between the terminals of the piezoelectric cell.

It is worth noting that for the electrostatic devices the damping force, being voltage controlled, can be dynamically optimized straightforwardly. For the other transducer types the approach to dynamic damping control is less straightforward. Since most likely motion scavenging applications will operate with highly variable source characteristics (i.e. frequency and amplitude), this dynamic possibility is crucial. Many reported devices have been tested with fixed sources, where this issue is not directly addressed. The proposed circuit in section 3 below, for electromagnetic transduction, includes a dynamic optimization capability.

The purpose of the power electronics circuitry, then, is two-fold, as shown in Figure 1, i.e. to regulate the power supply rail for the load electronics by extracting power from the transducer, and also to keep the transducer operating with the damping force that achieves the highest power density. Each of the three damper types presents different challenges in the design of the power electronics, and these add to the trade-offs in system design. Below we examine these issues for each of the three transducer types in turn.

\section{ELECTROMAGNETIC GENERATORS}

The common implementation of the electromagnetic resonant generator uses a permanent magnet and coil arrangement to provide the damping. Such a generator can also be termed a velocity damped resonant generator (VDRG), because the damping force is proportional to (and opposing) the proof mass internal velocity. This style of generator is best suited to higher frequency, low amplitude vibration sources. An illustrative case is a source vibration with an amplitude $\mathrm{Y}_{\mathrm{o}}=25 \mu \mathrm{m}$ at a frequency $\mathrm{f}=322 \mathrm{~Hz}$, used to drive a VDRG with an internal displacement limit $Z_{1}=1 \mathrm{~mm}$ and proof mass $\mathrm{m}=$ $0.5 \mathrm{~g}$. This follows the example in (El-hami et al., 2001). If we assume that the system has been tuned to operate at the resonant point, the optimal damping (for maximum power extraction) will be that which just allows the proof mass to move to its displacement limits. Thus in this case the resonant system must provide a displacement gain of 40. The optimal damping factor will be given by:

$$
\zeta=\frac{1}{2} \frac{Y_{0}}{Z_{l}}
$$

So in this case $\zeta=0.05$, a very lightly damped system.

The power extracted by this optimal damper is given by (Mitcheson et al., 2004a):

$$
P_{D}^{O p t}=\frac{1}{2} v^{2} D=\frac{1}{2}\left(\omega Z_{l}\right)^{2}\left(2 m \omega_{n} \zeta\right)
$$

where $\mathrm{v}$ is the proof mass velocity, $\mathrm{D}$ the damping coefficient, $\omega=2 \pi \mathrm{f}$, and $\omega_{\mathrm{n}}$ is the resonance frequency. For the given parameter values we obtain a power of 48.7 $\mathrm{mW}$.

A key design choice for the power processing is the voltage (and corresponding current) at which this power will be extracted. Conventional switch-mode circuits that include diodes must work at well above $1 \mathrm{~V}$ in order for the conduction power loss in the diode to be relatively small. Even with synchronous rectification with a MOSFET, it would be advantageous to operate at a relatively high voltage and low current. The counterinfluence is that a large active conductor length is required in the coil to achieve high voltages, and the coil can become difficult to fabricate or its size may exceed the available space. Using a large number of turns increases the induced voltage proportionately but also increases the self inductance of the coil at something close to the square of the number of turns. A high inductance requires a long conduction period to reach the value of current corresponding to optimal damping, and this can lead to high resistive losses. Adding more conductor material to the coil (more turns of the same cross section or the same turns at greater cross section) increases the area or the length over which flux must be supported in the air gap between the magnetic materials and requires a larger volume of permanent magnet.

For a coil with an active length of $l_{a}$ (the length that cuts the magnetic field during vibration) and a number of 


\section{P. D. Mitcheson, T. C. Green and E. M. Yeatman, "Power Processing Circuits ..."}

turns $N$, the voltage induced in the generator is: $V_{G}=N B l_{a}\left(\omega Z_{l}\right)$. The maximum flux density likely to be realised in the VDRG is about $1.2 \mathrm{~T}$. For a microengineered generator, an active length of $20 \mathrm{~mm}$ might be possible. This gives an induced voltage per turn of 48 $\mathrm{mV}$. It is clear that if a single turn is used then very low circuit impedances will be necessary to achieve the $2 \mathrm{~A}$ peak current required to extract $48 \mathrm{~mW}$. As a first estimate it was considered that up to 6 turns would be feasible and voltages up to $300 \mathrm{mV}$ might be achieved. This voltage needs rectification, but is clearly too low for the use of conventional diode rectifiers. The voltage also needs to be stepped up by a ratio of about 10 for use in standard electronics.

The case examined here, although realistic, is a specific and arbitrary one, and it could be argued that increasing $\mathrm{N}$ is feasible, and would greatly ease the difficulties in achieving efficient conversion and regulation. However, the required $\mathrm{N}$ could easily be much greater in other practical cases, where the flux gradient, active length and/or operating frequency is lower, and the literature indicates that high output voltages can often not be achieved. Thus we believe that the low-voltage rectification and step-up requirements are general to a large fraction of electromagnetic inertial microgenerators.

\subsection{Proposed Dual Polarity Boost Circuit}

Our proposed solution is to separately process the positive and negative half cycles of the generated voltage. Diode rectification is replaced by alternate activation of one of two voltage boost circuits. This is a form of synchronous rectification which avoids a series connection of separate rectifier and voltage converter. To limit the step-up ratio, the two circuits will provide half the output voltage each. The target output voltage is $3.3 \mathrm{~V}$ and this will be provided as $\pm 1.65 \mathrm{~V}$.

Figure 2 shows the two boost converter sub-circuits: one configured to produce the top half of the output voltage when the generator voltage is positive, and one configured to produce the lower half, when the generator voltage is negative. Because the generator voltage is small it is not able to forward bias the parasitic diodes of the MOSFETS. This means that in order to prevent conduction in the negative polarity boost converter when the generator voltage is positive, it is sufficient to hold off the MOSFET of the negative polarity converter. This gating of the two converters needs to be synchronised to the generator voltage. Synchronous rectification has been integrated into the boost converter so as to avoid series connection of separate rectifier and boost stages.

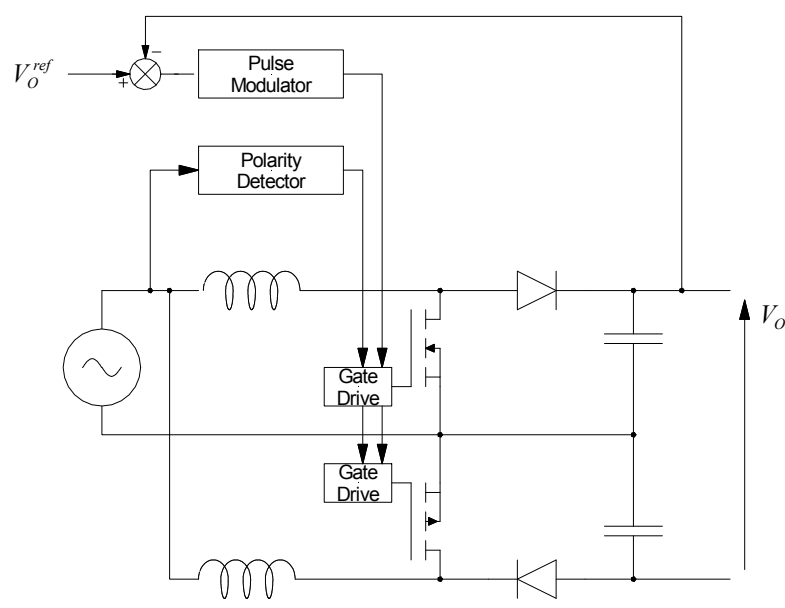

Figure 2. A dual polarity boost converter.

It is proposed to operate the boost converters in discontinuous conduction mode to avoid turn-on power loss in the MOSFET and reverse recovery effects in the diode. Several other benefits follow from this choice: relatively small passive components can be employed and a degree of resonant action can be added to the output side to manage the device parasitic capacitance. Schottky diodes have been used in this simple example but synchronously switched MOSFETs could be used instead.

The generator was modelled with 4 rectangular turns of $20 \mathrm{~mm}$ by $4 \mathrm{~mm}$ using $0.4 \mathrm{~mm}$ radius wire. The generator parameters are then: peak voltage $95 \mathrm{mV}$, self inductance $370 \mathrm{nH}$, resistance $7 \mathrm{~m} \Omega$, capacitance $7 \mathrm{pF}$. The main inductor was a Brooks coil of 6 turns of $0.6 \mathrm{~mm}$ square section wire giving: inductance $1.5 \mu \mathrm{H}$, resistance $28 \mathrm{~m} \Omega$ and capacitance $31 \mathrm{pF}$. The MOSFET model was based on the commercial 2 N6660 but with an area scaled by a factor of 16 (and the bonding wire resistance reduced). 
P. D. Mitcheson, T. C. Green and E. M. Yeatman, "Power Processing Circuits ..."
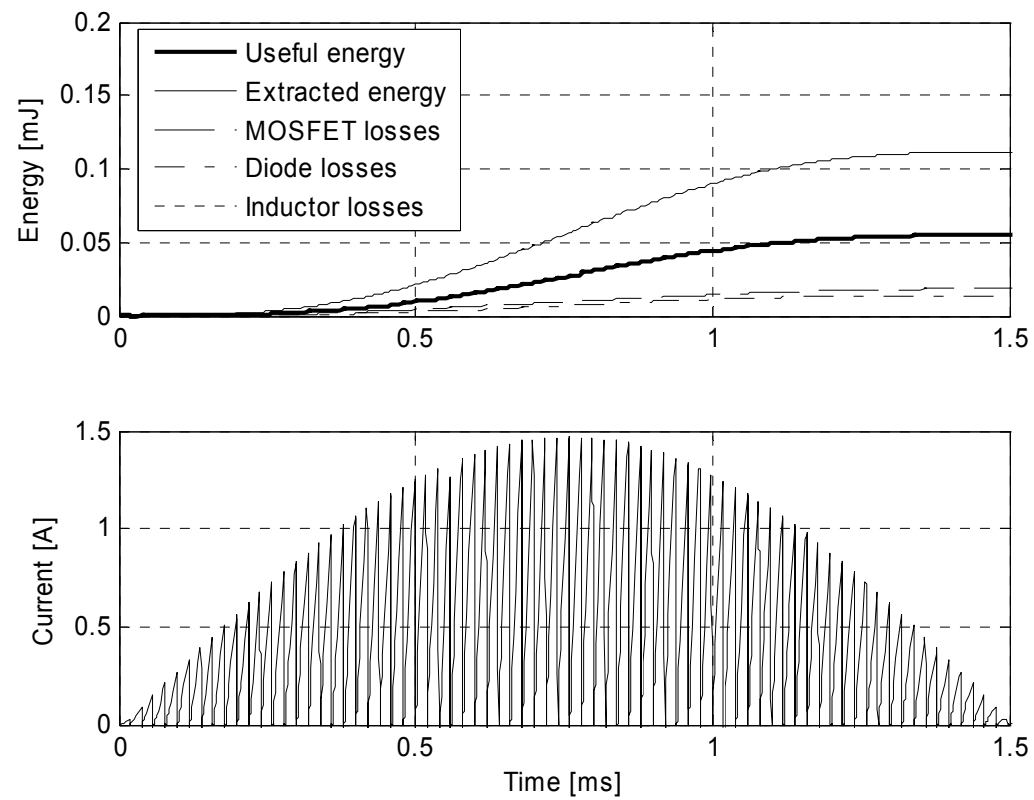

Figure 3. Spice simulation of the circuit of Fig. 2, positive half cycle, for a source displacement of $25 \mu \mathrm{m}$ at $322 \mathrm{~Hz}$. Top: accumulated energy extracted from the coil, output to the reservoir, and dissipated in the three main loss mechanisms. The three loss mechanism lines are of similar magnitude. Bottom: boost inductor instantaneous current.
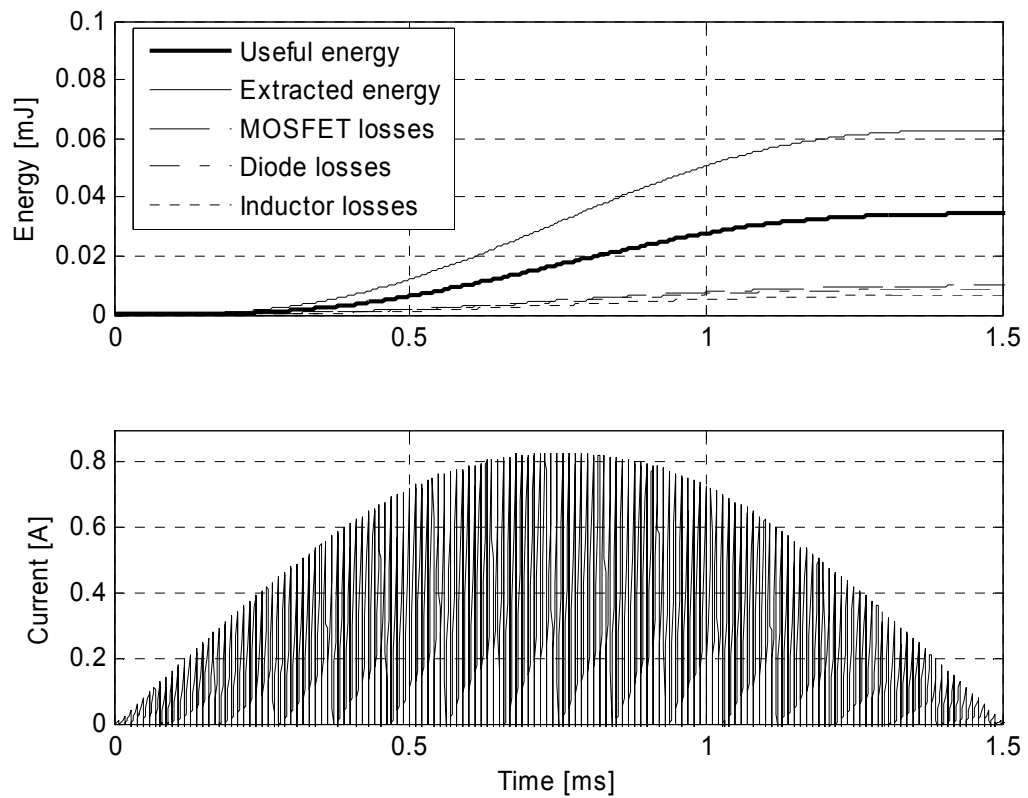

Figure 4. Spice simulation as in Fig. 3, for a reduced source displacement of $12.5 \mu \mathrm{m}$.

Figure 3 shows the results of a spice simulation of operation over the positive half-cycle. The MOSFET was switched at $50 \mathrm{kHz}$ with an on-time of $18 \mu$ s. The lower graph shows that the current drawn from the generator follows a sinusoidal envelope and the cycle-by-cycle of the current pulses reaches a peak of approximately 1.5 A.
This represents $49 \mathrm{~mW}$ taken from the $195 \mathrm{mV}$ source. The top axes show cumulative energies: input, output, resistance power losses, MOSFET power loss and diode power loss. The ratio of output energy to input energy indicates that the converter is operating at about $44 \%$ 


\section{P. D. Mitcheson, T. C. Green and E. M. Yeatman, "Power Processing Circuits ..."}

efficiency and that power loss in the MOSFET is the largest cause of inefficiency.

As stated above, the size of the MOSFET used in this example has been scaled up from that of a commercial device by 16 times. As can be seen from the sensitivity analysis presented in Table 1, increasing the MOSFET channel width further does not improve the converter efficiency. This is because the resistance of the coil and the inductor of the boost converter dominate the efficiency, although a decrease in width by a factor of two pushes the efficiency down to 0.38 as the MOSFET losses become more important.

Table 1: Effect of MOSFET size on system effectiveness

\begin{tabular}{|c|c|}
\hline $\begin{array}{c}\text { MOSFET width (as fraction } \\
\text { of standard 2660N width) }\end{array}$ & System effectiveness \\
\hline 8 & 0.38 \\
\hline 16 & 0.44 \\
\hline 32 & 0.5 \\
\hline
\end{tabular}

In Fig. 4, the same power processing circuit is used to provide the optimal damping factor under a different operating condition, whilst still maintaining the same efficiency. In this case, the amplitude of the input motion is half that of the previous example which, for the same generator and coil dimensions, halves the power which can be extracted under optimal conditions. With the same coil configuration, the voltage generated in the coil is unchanged (the peak relative velocity between the magnet and coil is the same as the first example), and so the current drawn must be halved. This is achieved by halving the on-time of the MOSFET in the boost converter.

As can be seen, the average current is half of that in the previous example, and the effectiveness of the system is almost unchanged. The process of tuning the damping factor in this way can easily be achieved on line by altering the gate signals to the MOSFETs in the converter.

These results show that for low voltage electromagnetic generators with output powers in the region of $50 \mathrm{~mW}$, it is possible to achieve up-conversion to useful voltages with an efficiency in the region of $50 \%$.

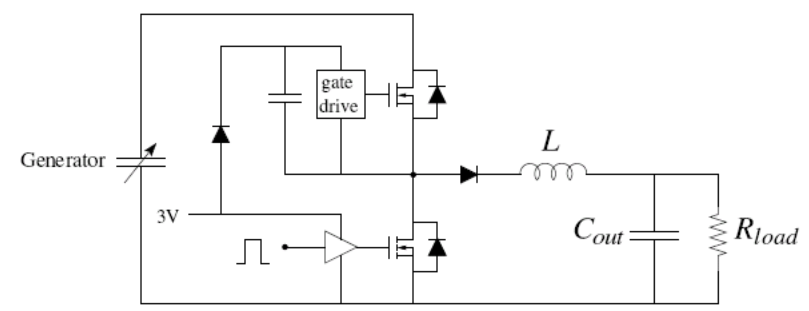

Figure 5. Modified buck converter.
The circuit has been simulated with the dominant parasitic components accounted for and with reasonable device models.

\section{ELECTROSTATIC GENERATORS}

The fundamental cause of difficulty in processing the output power for constant-charge electrostatic micro generators is that they work with small amounts of charge at high voltage. The principle of operation is that a variable capacitor is charged to a relatively low voltage at high capacitance. The optimal value of the pre-charging voltage is dictated by the operating condition and architecture of generator (Mitcheson et al 2004). When the generator experiences acceleration, the capacitance of the variable capacitor falls and, assuming the plates are electrically isolated as they separate, the voltage rises. Under typical operation, the voltage generated on the plates can be of the order of a few hundred volts. This charge must be down-converted to a lower voltage in order to be suitable for powering low-power, low-voltage loads.

The energy generated is given by:

$$
E=\frac{1}{2} Q^{2}\left[\frac{1}{C_{\text {open }}}-\frac{1}{C_{\text {closed }}}\right]
$$

and thus for a given amount of charge it is necessary to achieve a high ratio between the open and closed capacitance in order to maximize energy generation. Parasitic capacitance in parallel with the generator is a major problem. Such parallel parasitic capacitance is likely to be small compared to the maximum capacitance of the generator, but it will generally be substantial compared to the minimum (open) generator capacitance, and will therefore adversely affect power generation. Therefore one of the main challenges for the circuit design task is to minimize the parasitic capacitance connected to the generator.

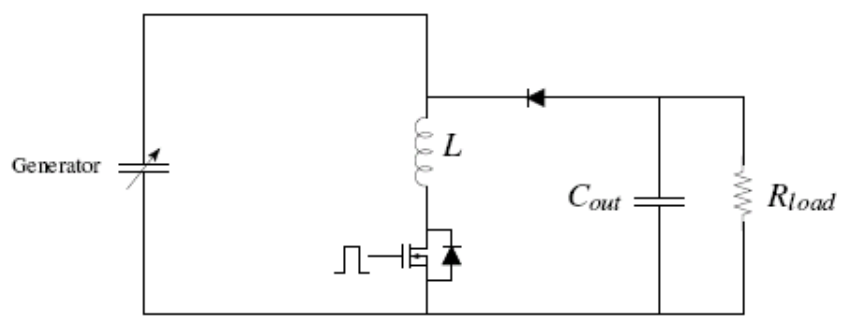

Figure 6. Modified flyback converter. 


\section{P. D. Mitcheson, T. C. Green and E. M. Yeatman, "Power Processing Circuits ..."}

The circuit of Fig. 5 shows a buck converter circuit which has previously been simulated using the Silvaco finite element device simulator (Stark et al., 2006) using custom designed semiconductor devices rated for high voltage blocking (around $250 \mathrm{~V}$ ), low off-state leakage and low junction capacitances. This circuit was initially investigated because it appears to be the simplest method of down-converting the high voltage on the generator. The simulations in Silvaco allowed the conversion efficiency of the converter to be evaluated using a mixedmode finite element/lumped element simulation for the devices and passive components respectively. The overall effectiveness of a micro-generator is more complex than just the efficiency of the power processing circuitry, and has been defined as a product of several terms (Stark et al., 2006). Two of the most important are the generation efficiency $n_{g e n}$ and the conversion efficiency $n_{\text {conv }}$. These terms are defined for the constant charge mode, electrostatic case as follows:

$$
\begin{gathered}
n_{\text {gen }}=\frac{E_{\text {open }}}{W_{\text {field }}+E_{\text {closed }}} \\
n_{\text {conv }}=\frac{E_{\text {out }}}{E_{\text {open }}}
\end{gathered}
$$

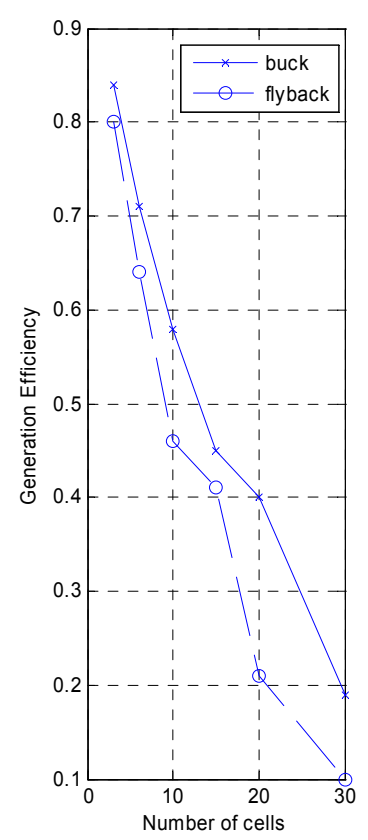

where $E_{\text {open }}$ is the energy stored on the moving plate capacitor at minimum capacitance, $E_{\text {closed }}$ the priming energy on the capacitor at maximum capacitance, $W_{\text {field }}$ is the amount of work that could have been done against the electric field as the plates separate and $E_{\text {out }}$ is the energy available after processing by the converter.

In the buck converter circuit the depletion layer capacitance of the blocking junction of the high-side MOSFET forms a parasitic capacitance in parallel with the generator capacitor as the generator voltage rises. Energy stored in this depletion layer capacitance is lost when the MOSFET is turned on.

Parasitic capacitance in parallel with the generation capacitor will reduce $n_{g e n}$, and switching and conduction losses in the converter will reduce $n_{\text {conv }}$. Increasing the cross sectional area of the MOSFET and diode will tend to increase $n_{\text {conv }}$ but decrease $n_{\text {gen }}$, because of the associated additional parasitic capacitance.

Models of the custom designed semiconductor devices were created in PSpice so that $n_{\text {conv }}$ and $n_{\text {gen }}$ could be simultaneously evaluated. The results are shown in Fig. 7 where the generator efficiency is shown as the product of $n_{g e n}$ and $n_{c o n v}$. The number of cells refers to the number of $0.015 \mathrm{~mm}^{2}$ cells that were used for the MOSFET and the diode. As can be seen, increasing the number of cells increases the conversion efficiency but reduces the generation efficiency. From this preliminary study, ten cells appears to give the highest overall generation efficiency.

Figure 7. Effectiveness and efficiencies of an electrostatic micro-generator. 


\section{P. D. Mitcheson, T. C. Green and E. M. Yeatman, "Power Processing Circuits ..."}

An additional energy loss mechanism associated with the buck converter circuit is a shoot-through current which reverse biases the blocking junction in the low side MOSFET. The high-side gate drive is also non-trivial to design. A possible solution to these two problems is to use a modified version of the flyback converter, shown in Fig. 6. An isolated flyback converter has been suggested in (Despesse, 2005), although details of the device parasitics are not presented. However, when this circuit was evaluated in PSpice, although the conversion efficiency of the flyback converter can be higher at higher cross sectional areas of device (because of the lack of shoot-through current), the additional parasitic capacitance from the diode reduces the generation efficiency too quickly, and overall, the buck converter achieves a higher efficiency.

\section{PIEZOELECTRIC GENERATORS}

Piezoelectric devices are attractive from a power processing point of view, as they can produce voltages in some practical micro-generator applications (Roundy et al., 2003) which can be processed with off the shelf semiconductor devices. However, obtaining a high damping force can be difficult with piezoelectric devices operating at low frequency due to internal leakage, and to limitations on the practical geometry and dimensions, most such devices using bimorph cantilevers. Unlike the electromagnetic and electrostatic cases, for piezoelectric generators a number of authors have described power processing circuits for converting the output to useful form.

For example, Ottman et al. designed an optimised power processing circuit for a piezoelectric transducer (Ottman et al., 2002). In this case relatively large voltages were obtained from the transducer (up to $100 \mathrm{~V}$ ), so that full-wave diode rectification was practical, followed by a conventional DC-DC step-down convertor. The use of duty cycle to vary the damping factor on the transducer was demonstrated.

Roundy (Roundy et al., 2003) and Ottman (Ottman et al., 2002) have both shown that piezoelectric generators can achieve higher power densities when driving resistive loads than when they are connected to a simple power supply consisting of a bridge rectifier and smoothing capacitor. We have shown that the same is true for electromagnetic devices (Mitcheson et al., 2004a), and the circuit presented in section 2 satisfies this requirement.

There will be cases where piezoelectric generators produce insufficiently high voltages for straightforward rectification, so that voltage boosting circuits will be a useful approach to efficient rectification. However, this is not the only motivation for voltage boost circuits. The output impedance of piezoelectric cells is typically dominated by the cell capacitance, so that a reasonable equivalent circuit for the piezoelectric source is an ideal current source in parallel with this capacitance $\mathrm{C}_{\mathrm{o}}$. In such a case, the optimal load resistance $\mathrm{R}$ for a real load is simply $R=1 / \omega C_{0}$, with $\omega$ the source frequency. The extracted power in this case is typically far below what can theoretically be extracted: this theoretical output power is limited by the real part of the output impedance of the piezo-cell, and is only accessible (in a linear circuit) if the output capacitance is compensated out by the load reactance.

One approach to raising the extracted power closer to this theoretical limit is presented in (Guyomar et al., 2005). Here the output voltage is increased by synchronously switching the charge on the piezo-cell, at its peak, into an inductor. This has the effect of increasing the optimum load resistance, as would be the case if the output reactance was compensated, and so significantly increasing the extracted power.

\section{CONCLUSIONS}

Electromagnetic and constant-charge electrostatic inertial generators each present significant challenges for output conversion and regulation, because of low and high output voltages respectively, and the need to achieve high efficiency in both cases despite high sensitivity to parasitics. We have presented circuit topologies for both these cases, and simulated them with realistic device models. In both cases acceptable efficiencies could be obtained. Since the proposed circuits each allow the effective load on the generator to be varied using switching duty cycles, they also present a convenient mechanism for dynamically optimising the load to extract maximum power under varying source motion.

\section{REFERENCES}

Despesse, G., Jager, T., Basrour, S., Chaillout, J. J., Charlot, B. Leger, J. M., Vassilvev, A. (2005) In DTIP.

El-hami, M., Glynne-Jones, P., White, N. M., Hill, M., Beeby, S., James, E., Brown, A. D. and Ross, J. N. (2001) Sensors and Actuators A: Physical, 92, 335342.

Guyomar, D., Badel, A., Lefeuvre, E. and Richard, C. (2005) Ieee Transactions on Ultrasonics Ferroelectrics and Frequency Control, 52, 584-595.

Meninger, S., Mur-Miranda, J. O., Amirtharajah, R., Chandrakasan, A. and Lang, J. H. (2001) Very Large Scale Integration (VLSI) Systems, IEEE Transactions on, 9, 64-76. 
P. D. Mitcheson, T. C. Green and E. M. Yeatman, "Power Processing Circuits ..."

Mitcheson, P. D., Green, T. C., Yeatman, E. M. and Holmes, A. S. (2004a) Microelectromechanical Systems, Journal of, 13, 429-440.

Mitcheson, P. D., Miao, P., Stark, B. H., Yeatman, E. M., Holmes, A. S. and Green, T. C. (2004b) Sensors and Actuators A: Physical, 115, 523-529.

Ottman, G. K., Hofmann, H. F., Bhatt, A. C. and Lesieutre, G. A. (2002) Power Electronics, IEEE Transactions on, 17, 669-676.
Roundy, S., Wright, P. K. and Rabaey, J. M. (2003) Energy Scavenging for Wireless Sensor Networks, Kluwer Academic Publishers, Boston, Massachusetts. Stark, B. H., Mitcheson, P. D., Miao, P., Green, T. C., Yeatman, E. M. and Holmes, A. S. (2006) Power Electronics, IEEE Transactions on, 21, 27-37.

Williams, C. B. and Yates, R. B. (1995) In Solid-State Sensors and Actuators, 1995 and Eurosensors IX. Transducers '95. The 8th International Conference on, Vol. 1, pp. 369-372. 